\title{
The Influence of Instruction on Color/Form Classification Strategies and Longterm Memory: A Developmental Study \\ Dennis Febr
}

Methods children use to categorize visual stimuli offer a starting point in the investigation of the psychological effects works of art have on individuals. Children's use of color or form as classification dimensions is one focus of categorization studies. Such studies seek to define the processes involved in classifying visual stimuli. Employing this approach, this study was designed to clarify the role of development and the influence of instruction on children's performance in classifying nonrepresentational paintings.

Specifically, this study was undertaken to determine the effects of instruction on performance of a color/form classification task, and to examine the effects of time (a 2-week period) on retention of the instructed material. Additionally, performance differences between three developmental levels (grades 1,4 , and 7 ) were observed. This study casts light on the issue of which components of paintings are attended to by different developmental levels, building on the work of Hill and Kuiken (1975), Hardiman and Zernich (1982), and Phillips (1985).

The following hypotheses were tested:

Hypothesis I. Untrained (i.e., control group) seventh graders will classify novel stimuli along the form dimension significantly more than will 
untrained fourth graders, who in turn will classify along the form dimension significantly more than will untrained first graders.

Hypothesis II. Following instruction, the color-trained group at each age level will classify novel stimuli along the color dimension rather than the form dimension to a degree significantly greater than chance.

Hypothesis III. Following instruction, the form-instructed group at each age level will classify novel stimuli along the form dimension to a degree significantly greater than chance.

Hypothesis IV. The post-test (given two weeks after the instruction sessions) will indicate significant retention of respective instructions within trained groups.

\section{Method}

\section{Subjects}

One hundred and sixty-five subjects representing 3 developmental levels ( 57 first graders, 50 fourth graders, and 58 seventh graders) were selected. Each group was divided into three subgroups. One subgroup from each level was given color-selection training; another was given formselection training, and the third functioned as a control. All subjects were randomly assigned to groups. The 3 groups at each level were given the same color/form classification task (using 9 triadic sets) as a pretest. Then one group at each level received form discrimination instruction, one group received color discrimination instruction, and one group received no instruction.

Working Papers in Art Education 1988 
Immediately following its instruction session, each group was given classification posttests using 9 novel triadic sets. The control group was given the same posttest. Two weeks later, all groups were given a third set of novel stimuli for a second posttest. Consequently, this was a repeated measures design with each subject nested by grade and group, and crossed over time.

\section{Stimuli}

The stimuli used in this study were color slides of nonrepresentational Western masterworks of the twentieth century as judged by acknowledged art historians (cf. Jansen, 1971; Arnason, 1982; Gombrich, 1972). These stimuli meet the definition posed by Medin and Schaffer (1978) of ill-defined categories: "stimuli for which clearcut defining attributes and obvious classification rules do not exist. Yet on exposure to a large number of instances of an ill-defined category, classification is possible, although the rules used may be difficult to verbalize." The slides were selected such that the subjects were presented with triadic sets containing the following:

A. Two paintings that matched in color. These two paintings were markedly different in form (here defined as the formal elements of painting, e.g., line, composition, texture).

B. One of the two color-matched paintings matched the form of the third member of the triad, but the color of the third member was markedly different from the color of the other two. 
The mature works of artists such as Olitski, Reinhardt, Pollock, Rothko, Poussett-Dart, Motherwell, and Stella were used because of their absence of subject matter.

In an effort to address the problem of inconsistency in research in visual art perception, masterworks easily available to any researcher (as opposed to idiosyncratic stimuli) were used in the hope that such stimuli will become a base common to future research in this area.

\section{Results}

\section{Color-trained Groups}

Pretest preferences of all levels were for form (grade $1-64.0 \%$; grade $4-47.5 \%$, a plurality; grade $7-65.0 \%$ ). Following training, preferences were overwhelmingly for color in Posttest I (grade 1 - 88.3\%; grade 4 - $75.6 \%$; grade 7 - $88.8 \%$ ) and Posttest II, although slightly less so for Posttest II (grade 1 - 62.1\%; grade 4 - 52.8\%; grade 7 - $73.3 \%$ ).

Goodman's Likelihood Ratio Chi-Square Test indicated that increases from the Pretest to Posttest I were significant for all grade levels (p > .0001). Increases from the Pretest to Posttest II were significant for the Grades 1 and 4 comparison (p. > .0002), as well as for the Grades 1 and 7 comparison ( $\underline{p}>.0001)$ and the Grades 4 and 7 comparison $(\underline{p}>.0001)$.

\section{Form-trained Groups}

Pretest preferences at all levels were for form (grade 1 - 58.7\%; grade $4-67.2 \%$; grade $7-66.3 \%)$. following training, preferences were overwhelmingly for form in both Posttest I (grade 1 - $86.4 \%$; grade 4 - 
95.7\%; grade 7 - 95.7\%) and Posttest II (grade 1 - 77.5\%; grade 4 - 72.8\%; grade $7-90.5 \%)$.

Goodman's Likelihood Ratio Chi-square Test measured posttraining increases in form choices for the form-trained groups. Increases from the Pretest to Posttest II were significant for all levels (․ . 0001). Increases from the Pretest to Posttest II were significant for the Grades 1 and 4 comparison ( $\mathrm{p}>.0074)$ and for the Grades 1 and 7 comparison ( $\mathrm{p}>.0001)$, but not for the Grades 4 and 7 comparison ( $\mathfrak{p}>.0529)$. The contrast in Pretest to Posttest II results between color-trained groups and form-trained groups is accounted for by the fact that Pretest form choices were much higher for both groups than were Pretest color choices.

\section{Control Groups}

All levels preferred form in the Pretest (grade $1-46.9 \%$, a plurality; grade 4 - 62.2\%; grade 7 - 64.8\%), Posttest I (grade 1 - $54.4 \%$; grade 4 57.9\%; grade 7 - 76.6\%), and Posttest II (grade 1 - 42.7\%, a plurality; grade 4 - 54.0\%; grade $7-74.7 \%$ ). A developmental trend for form was indicated in all three time periods.

Goodman's Likelihood Ratio Chi-square Test measured noncolor preference across all three tests. Noncolor preference was significant at all levels $(\underline{p}>.0001)$. The Chi-square also measured the developmental effect for noncolor choices. Grade 4 preferred noncolor more than grade 1 did ( $\mathrm{p}>.0383$ ), and grade 7 preferred noncolor more than grade 4 did $(\underline{p}>.0028)$. 
Color groups' Pretest color choices were $32.9 \%$. Posttest I and II scores jumped to 84.2 and $62.7 \%$ respectively. Their form choices dropped from 57.9 to $6.8 \%$, and climbed to $31.2 \%$. (Unmatched choices accounted for $9.2,9.0$, and $6.1 \%)$

Form groups' Pretest color choices were $29.3 \%$. Posttest I and II scores dropped to 4.6 and $15.1 \%$ respectively. Their form choices climbed from $64.1 \%$ to 92.5 and $80.3 \%$. (Unmatched choices accounted for 6.6 , 2.9, and $7.0 \%$.) Control groups' Pretest color choices were $33.9 \%$. Posttest I and II scores were 27.5 and $24.5 \%$ respectively. Their form choices were $60.0,57.1$, and $62.9 \%$. (Unmatched choices accounted for $8.1,15.4$, and $12.4 \%$.)

A linear model analysis of color-matched responses for all grades, groups, and times indicated that differences in responses between groups were significant ( $\underline{p}>.0001)$, as were differences in responses across times $(\underline{p}>.0087)$. The two-way interaction of response differences between groups across times was significant ( $\underline{p}>.0001)$, as was the three-way interaction of grade levels between groups across times $(\underline{p}>.001)$.

The results of a linear model analysis of form-matched responses for all grades, groups, and times indicated that response differences between groups were significant $(\underline{p}>.0001)$, as were response differences across times $(\underline{p}>.0001)$. The two-way interaction of grade levels across times was significant ( $\underline{p}>.0046)$, as was the two-way interaction of groups across times. The three-way interaction of grade levels between groups across times was significant $(\underline{p}>.0203)$.

Working Papers in Art Education 1988 
Unlike the univariate ANOVA, the multivariate ANOVA makes no assumptions about covariance of repeated measures. For this reason, the data were analyzed with a linear model manova. It indicated a significant Pretest interaction between grade and group ( $\underline{p}>.0092)$. Posttest I results indicated significance for group ( $\underline{p}>.0001$ ) and the interaction between grade and group ( $\underline{p}>.0271)$. Posttest II results indicated significance for group ( $\mathrm{p}>.0001)$. Between subjects effects were significant for group $(\underline{p}>.0001)$. Within subjects effects were significant for time $(\underline{p}>.0001)$, and the two-way interaction between time and group ( $\underline{p}>.0001)$. The three-way interaction between time, grade, and group was significant as well $(\mathrm{p}>.0001)$.

\section{Discussion}

This developmental study was designed to investigate the influence of instruction on the color/form classification task and longterm memory. It was hypothesized that (a) there would be a developmental trend for form among untrained (i.e., control group) subjects; (b) color-trained groups would classify stimuli by color to a degree significantly greater than chance; (c) form-trained groups would classify stimuli by form to a degree significantly greater than chance; and (d) the posttest (given two weeks after the instruction sessions) will indicate significant retention of respective instructions within trained groups. Parametric and nonparametric measures supported Hypothesis I, that control group seventh graders will classify according to the form dimension more than 
dimension more than will control group first graders. All three grade levels classified more by form than color, but preference for form increased as age increased. This study produced evidence supporting a systematic developmental trend toward form as the prominent cue for classification. The findings of this study indicate a developmental trend for increasing use of form as a classification dimension; however, in this study even first graders slightly preferred form over color. Hypotheses II and III were supported by data suggesting that both color and form instruction were effective. Across grade levels, the data showed significant differences in responses strategies according to the type of instruction received.

Significant retention of instruction influence in longterm memory, Hypothesis IV, was confirmed at all developmental levels, and with both color-and form-trained groups. The effects of instruction diminished, however, over the two-week period; both color-and form-trained groups demonstrated a tendency to revert to pre-instruction performance levels.

\section{Conclusions}

The significance of this study lies in its approach to the traditional color/form classification task. Use of slides of contemporary masterworks of nonrepresentational painting (widely available analytic stimuli) place it specifically in the context of art education. Use of such easily available stimuli facilitates replication and general discussion, a much-needed development in research in this area.

This study supported the position of Hardiman and Zernich (1985) that, when controlling for the influence of subject matter as a classification

Working Papers in Art Education 1988 
cue, young children can sort paintings using a variety of perceptual cues. It was found that there is a developmental trend for increased use of form as a classification cue, although all levels preferred form over color. This study indicated that if there is a developmental level that prefers color, it occurs before grade 1 .

\section{References}

Arnason, H.H. (1982). History of modern art. New York: Harry N. Abrams, Inc.

Gombrich, E. H. (1966). The story of art. New York: Phaidon Publishers.

Hardiman, G. W., \& Zernich, T. (1982). The relative influence of parts and wholes in shaping preference responses to paintings. Studies in Art Education, 23, 3, 31-38.

Hardiman, G. W., \& Zernich, T. (1985). Discrimination of style in painting: A developmental study. Studies in Art Education, 26, 3, 157-162.

Hill, K., \& Kuiken, D. (1975). Conceptual complexity and concept learning of painting styles. Journal of Personality and Social Psychology, 32 , 1, 154-159.

Jansen, H. W. (1971). History of art. New York: Harry N. Abrams, Inc. Medin, C. L., \& Schaffer, M. M. (1978). Context theory of classification learning. Psychological Review, 3, 207-238.

Phillips, P. E. (1985). A developmental study of the selective viewing of paintings. Visual Arts Research, 11, 2. 